$\beta$ could be increased from 0.032 (with no net toroidal current) to 0.042 (with a toroidal current). This phenomenon can be seen in Fig. 6 in which is plotted the converged eigenvalues as a function of $\beta$ for three different values of $J$, the net toroidal current:

a) $J=+0.5$ : the externally applied imposed twist of the magnetic field is reduced and the plasma configuration as a result remains unstable to global, external modes with a toroidal mode number $n=1$ at $\beta$ values well below 0.01 ; b) $J=0$ : corresponds to the standard configuration $(\beta<0.032)$;

c) $J=-0.5$ : the twist of the magnetic field is increased which causes the resonant surface for the $m=1, n=1$ component (where $m$ is the poloidal mode number) of the mode structure to move from the vacuum region into a region of high magnetic shear within the plasma. This causes the $n=1 \mathrm{fa}$ mily of modes to become stabilized up to $\beta=0.05$. The $n=2$ family of modes imposes the limiting value at $\beta=$ 0.042 .

The latter is promising result because the effect can be realized by driving a small current near the plasma surface using well-known radio frequency current drive techniques. It also demonstrates that by taking into account new parameters one can adjust the stability of magnetic fusion devices.
In the future, new types of devices such as the Helias type of stellarators (Fig. 2) and racetrack tokamaks (Fig. 4) will be studied in more detail using supercomputers running the TERPSICHORE program in order to identify and understand regimes of stable operation which could lead to improved designs for the next generation of fusion experiments and reactors.

\section{Conclusions}

Using computer simulation techniques to study the stability behaviour of reactor-relevant controlled fusion experiments we have been able to obtain new insights into the underlying physics, notably:

- For tokamaks, a scaling law has been found that states that the maximum achievable plasma pressure, as given by $\beta$, the ratio of the plasma pressure to the confining magnetic field energy density, is proportional to the total current $J$, and not to $J^{2}$ as expected. Furthermore, altering shaping parameters such as the elongation or the triangularity of the cross-section can only lead to an increase in $\beta$ if the total current is increased at the same time. - For the proposed W7X stellarator, helically twisting the magnetic axis stabilizes the plasma configuration.

- For Oak Ridge's ATF stellarator, the addition of a small net toroidal current in the direction of the magnetic field (in order to enhance the twist of the field lines) increases $\beta$ from 0.032 to 0.042 . This result clearly contradicts the established theory that the stability properties of stellarators are deteriorated by induced net toroidal currents.

Supercomputer simulations therefore allow novel plasma configurations to be studied, an important feature since reactor-relevant fusion experiments are very expensive, and it will no longer be possible to build a fundamentally new type of device without having performed intensive stability computations beforehand.

\section{REFERENCES}

[1] Hirshman S.P., van Rij W.I. and Merkel P., Comput. Phys. Commun. 43 (1986) 143; Hirshman S.P. et al., idem. 87 (1990) 396. [2] Degtyarev L.M. and Medvedev S.Yu., idem. 43 (1986) 29; Grimm R.C. et al., Meth. Comput. Phys. 16 (1976) 253.

[3] Troyon F. et al., Plasma Phys. 26 (1984) 209.

[4] Gruber R. and Rappaz J., "Finite Element Methods in Linear Ideal MHD", Springer Series in Computational Physics (Springer Verlag, Heidelberg) 1985.

[5] Troyon F. and Gruber R., Phys. Lett. 110A (1985) 29

[6] Anderson D.V. et al., Proc. Joint Varenna - Lausanne Int. Workshop on the Theory of Fusion Plasmas (Editrice Compositori, Bologna) 1988.

[7] Schwab C., idem.

\title{
The TERPSICHORE Project
}

The modular structure of the TERPSICHORE computer program. Using the ASTRID programming platform, a common memory and a command language is shared between the the various modules that perform specific tasks such as mapping the MHD equilibrium, constructing the stability matrix elements, interpolating, etc.

TERPSICHORE is a high-speed, efficient computer package that was developed to study the three-dimensional (3-d) stability of plasma in reactor-relevant devices. It presently comprises six basic modules involving: the interface to the magnetohydrodynamic (MHD) equilibrium, reconstruction of the MHD equilibrium, mapping of the MHD equilibrium from the equilibrium coordinates to the stability coordinates, construction of the stability matrix elements, solution of the eigenvalues, and analysis and diagnostics of the results. It is intended to incorporate additional modules that will: generate an adaptive radial mesh by interpolating the equilibrium quantities; minimize the number of selected Fourier mode pairs by using an algorithm based on an expert system approach (this would replace the tedious manual selection of repetitive choices); add a penalty function integral contribution to the potential energy of the variational form (see Eq. 2, page $\mathrm{xx}$ ) in order to eliminate destabilized modes belonging to the stable continuous spectrum.

The overall modular structure of TERPSI-

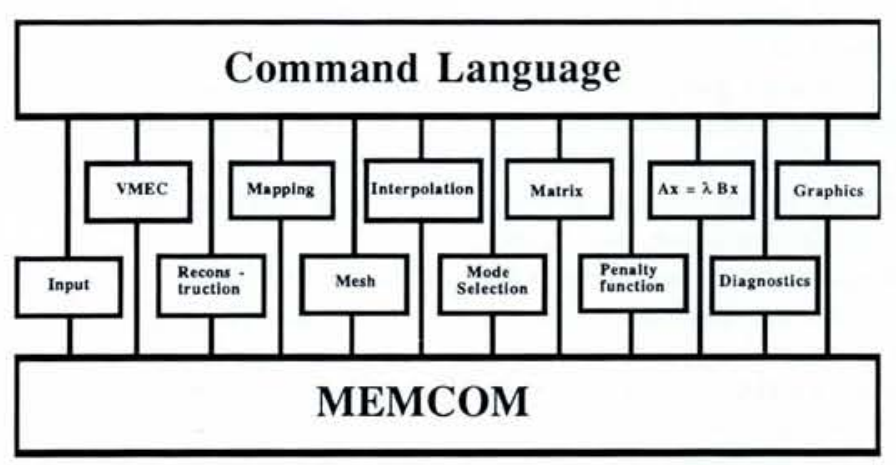

CHORE program, which is embedded in the ASTRID programming platform [Bonomi E. et al., GASOV Report 26 (EPFL, Lausanne) 1990], is shown in the figure above. The choice of very advanced numerical methods and a design which runs efficiently on vector-parallel computers with either shared or distributed memories (an operating speed of $2 \mathrm{Gflop} / \mathrm{s}$ has been achieved on the EPFL's Cray YMP8 machine - a feature that led to the team being awarded the Cray Gigaflop Performance Prize in 1989 and 1990 - have made TERPSICHORE an effective tool for plasma physicists. It enables the generation of significant results in a relatively short time crucial when studying 3-d devices where the parameter space is much wider than for 2-d geometries and many more cases need to be treated. For if we cannot simulate the 
various possibilities rapidly enough, computer experimentation becomes impractical.

\section{International Collaboration}

TERPSICHORE vividly demonstrates that an important program for supercomputer experimentation profits greatly from a collaboration between research scientists with complementary knowledge. The project was initiated at the Centre de Recherches en Physique des Plasmas (CRPP) of the Ecole Polytechnique Fédérale de Lausanne (EPFL) where specialists in MHD equilibria and stability were brought together for two years (1988-1989). The team included Dr. W. Anthony Cooper, a champion in vector analysis and the developer of the plasma and vacuum contributions in the variational form $\delta W$, Dr. Ulrich $H$. Schwenn from the Max-Planck Institut für Plasmaphysik (MPIPP), Garching bei München, Germany, who wrote the equilibrium-stability interface, $\mathrm{Dr}$. David V. Anderson from the Lawrence Livermore National Laboratory, USA, our specialist in vectorizing and parallelising the code, Dr. Ralf Gruber and Dr. Silvio Merazzi. The latter two scientists were mainly involved in the ASTRID aspects of the project, but they also contributed to the eigenvalue solver, the database management system, the command language and the graphics package.

The TERPSICHORE version for the Intel ipsc2 hypercube machine was prepared by Dr. Marc Gengler from the Computer Science Department of the EPFL. Dr. Guo Y. Fu is currently testing a new finite element approach and is adding terms to $\delta W$ which which will enable us to compute in a realistic way how the unstable solution moves away from equilibrium. In addition, we were able to benefit from many important interactions with Dr. S.P. Hirshman of Oak Ridge National Laboratory, USA, and Drs. J. Nührenberg, C. Schwab and P. Merkel of the MP-IPP. An official cooperation between the CRPP and the Keldysh Institute, Moscow is being pursued to further mathematical understanding of some peculiarities of the ideal MHD spectrum, and to validate uniaxial 3-d equilibrium solutions.

The test phase of the project lasted approximately one year. During this period, results generated by TERPSICHORE were compared with available 2-d and 3-d results produced by existing programs. TERPSICHORE entered its production phase in May 1990 and owing to the efficiency of the program it was possible to produce relevant physics in a short time.

Many groups based in the USA, Japan, Australia, USSR and Europe have asked for copies of the code in order to interpret their experimental measurements, design new experiments or to identify new magnetic confinement configurations. We have proposed an international collaboration between the interested groups whose aim would be direct the further development of TERPSICHORE and its application. It is also proposed to organize special IAEA workshops to discuss results.

R. Gruber, EPFL, Lausanne

\section{EUROGAM}

\section{New Dimensions to Studies of Nuclear Structure}

\author{
F.A. Beck \\ CRN Strasbourg, France
}

The combination of heavy ions and high resolution $\gamma$-ray multidetectors, comprising germanium elements surrounded by anti-Compton scintillator shields, has permitted this last decade a quantitative leap in the way we approach an understanding of the microscopic properties of the nucleus.

The product of the reaction of heavy ions is a compound nucleus with a very high angular momentum. A few particles then evaporate to give a low temperature, high angular momentum state that deexcites by radiating $\gamma$-rays (the so-called heavy ion fusion evaporation reaction).

The improved understanding applies not only to the individual excitations of nucleons but also to the collective behaviour of the nucleus. Perphaps the most spectacular discoveries are those providing evidence for very regular cascades of $\gamma$-ray emissions (some 15 to 20 transitions). This new type of $\gamma$-ray transition "band" was discovered at Daresbury Laboratory in the UK using ${ }^{152} D y$ nuclei. It was then more widely observed in the rare earth range (atomic number $A=130$ to $150)$ as well as in the range $A=190$. Microscopic calculations permit an interpretation based on a second stable minimum for the nuclear potential corresponding to a very deformed ("superdeformed") ellipsoidal nucleus with ratios of the major to minor axes approaching 1.6 for $A=130$ and 190 and approaching 2 in the region of $A=150$ (see Europhysics News 21 (1990) 86).

The structure of the superdeformed bands revealed unexpected features when compared with what had been established previously. There was found to be feeding below an angular momentum of $56 \hbar$ with rapid saturation; and de-excitation at spins around $22 \hbar$ within 1 to 3 transitions by a process which has not yet been revealed experimentally (because instruments are probably not sufficiently sensitive). The intensity of the superdeformed bands (between 1 and $2 \%$ relative to

Dr. F.A. Beck is Head of the Division de Physique Nucléaire, Centre de Recherches Nucléaires, F-67037 Strasbourg. He is responsible for the French participation in the EUROGAM collaboration and he became the Chairman of the EUROGAM Project Scientific Committee on 1 March 1991.

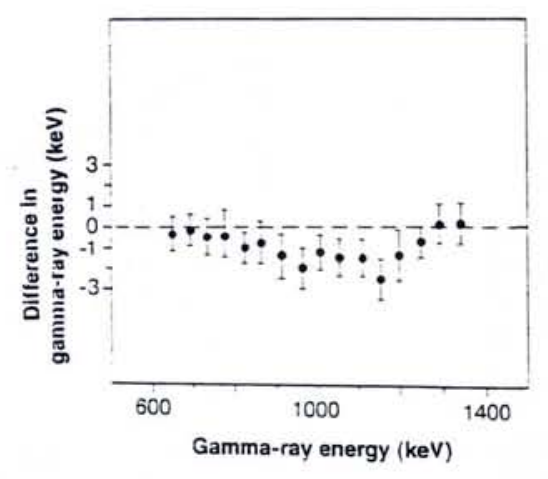

Fig. 1 - Differences in $\gamma$-ray energies in superdeformed bands between the yrast band in ${ }^{152} \mathrm{Dy}$ and the excited band in ${ }^{151} \mathrm{~Tb}$

the total reaction channel) also has a similar magnitude for the cases examined, except around $A=130$ where examples of stronger feeding have been observed. Microscopic calculations also show that the behaviour of the moments of inertia, whose values are always below those for rigid-body rotation (this has not yet been accounted for) is closely related to the occupation of "intruder" orbitals with high angular momenta.

Excited superdeformed bands have been observed recently, also in the range of masses $A=150$ and $A=190$. Their intensities are even weaker (some fractions of a percent of the total intensity of the reaction channel) and they exhibit the same general characteristics as the yrast bands (bands corresponding to states with the lowest excitation energy for a given energy).

\section{"Identical" bands}

The most astonishing discovery in this area was made by a group from Strasbourg (T. Bryrski et al., Phys. Rev. Lett. 64 (1990) 1650). The analysis of data produced within the context of a French-UK collaboration revealed superdeformed bands whose $\gamma$-ray transition energies are the same to within one part in a thousand for the ${ }^{152} \mathrm{Dy}$ (yrast band) and ${ }^{151} \mathrm{~Tb}$ (excited band) nuclei (see Fig. 1) whereas differences an order of magnitude larger were expected. Other examples of this phenomena involving the observation of "identical" bands in different nuclei have since been found in the rare earth region and the 